\title{
Original Article (short paper) \\ Biomechanical Parameters in Children with Unilateral and Bilateral Clubfoot during Vertical Jumps
}

\author{
Renato José Soares \\ Alex Sandra O Cerqueira \\ Universidade de Taubaté, Taubaté, SP, Brasil \\ Luis Mochizuki \\ Julio Serrão \\ Universidade de São Paulo, São Paulo, SP, Brasil \\ João P Vilas-Boas \\ Faculdade de Desporto, CIFI2D e LABIOMEP, Universidade do Porto, Porto, Portugal \\ Alberto C. Amadio \\ Universidade de São Paulo, São Paulo, SP, Brasil
}

\begin{abstract}
Gait analysis may offer information to choose the best exercise-based clinical intervention for the children with clubfoot. However, other motor abilities are not commonly investigated. The aim of this research was to analyze the biomechanics of countermovement vertical jumping in clubfooted children who had undergone surgery. Fourteen children with idiopathic clubfoot were selected and the control group consisted of 11 children. Clubfooted children showed less dorsiflexion in the jump preparation phase. In the impulse phase, this group showed more knee flexion and less plantarflexion associated with less magnitude of vertical reaction force and less muscular activity in the gastrocnemius medialis. In the landing phase, for clubfoot group, we found high loading rate for the first peak of vertical force, less plantarflexion and more knee flexion. Understanding the biomechanical changes of vertical jump landing should assist in better targeting of physical and sporting activities of this population.
\end{abstract}

Keywords: congenital talipes equinovarus, clinical biomechanics, kinematics, electromyography

\section{Introduction}

Idiopathic clubfoot (CF) is the most prevalent congenital deformity in orthopedics. This deformity varies from 0.64 to 6.8 per 1,000 live births, with a fixation of the foot in adduction, supination, and varus ${ }^{1}$. Calcaneus, navicular, and cuboid are medially rotated in relation to talus and are held in adduction and inversion. Although the foot is supinated, the forefoot is pronated in relation to the rearfoot, causing cavus. In addition, the first metatarsal is more plantar flexed ${ }^{2}$. Without any intervention to correct those deformities, such an infant will face difficult functional conditions in future, including problems with walking. Therefore, treatment for $\mathrm{CF}$ must begin as soon as the deformities are found, and periodic examinations are needed due to recurrent or residual deformities, which may require new treatment ${ }^{3,4}$.

It is necessary to evaluate how ankle-foot deformities affect gait and other motor skills after intervention. Any therapeutic approach is going to leave residual changes with anatomical imbalances, which will affect a toddler's first steps. Unfortunately, clinical evaluations (subjective patient evaluation and imaging evaluation) are not enough to inform about the performance of lower limbs during locomotion. That information can be supplemented with motion analysis applied to locomotion.
Most studies about CF are focused on walking, but this is not the only way to move. Moreover, demands for internal forces to walk are small compared to the real potential of lower limbs. Conversely, jump demands many more internal forces to be performed. There is no information about motion analysis applied to jumps in children with $\mathrm{CF}$.

Motion analysis in congenital CF children is applied to understand the mechanics of that dysfunction, for the affected and unaffected $\operatorname{sides}^{2-5}$. To improve rehabilitation procedures for $\mathrm{CF}$, gait and other motor skills should be evaluated. For example, countermovement vertical jump (CVJ) is commonly used in recreational activities and contributes to better motor control. Besides, vertical jump performance is related to lower limb strength. The CVJ's start position is the upright standing position. The jumper performs the countermovement (knee flexion, hip flexion, and ankle dorsiflexion, followed by knee extension, hip extension, and ankle plantar flexion) before jumping vertically up off the ground $^{6}$. During landing, internal net joint forces are generated to control the body limbs and reduce the total body momentum ${ }^{7,8}$.

Foot and ankle joints are important to stabilize body balance and mechanical loads applied to the body right after landing 9 . Such a mechanism is not clear in children with CF. How is the motor coordination of the lower limbs of children with $\mathrm{CF}$ in functional activities such as a vertical jump? It is not clear 
whether children with CF can control ankle and hip joints the same way as children with typical development. In fact, we do not expect that they will move with similar kinematics and kinetics compared with children with typical development. Under different anatomical constrains, we expect that children with $\mathrm{CF}$ will show different coordination in joints and muscles. Thus, such differences in coordination will reflect in changes in ground reaction forces applied during jumping and landing. How coordinated are propulsion and landing phases during CVJ in children with CF? These answers will contribute to better understanding the mechanics of functional abilities that require variable levels of effort.

The aim of this study is to analyze the countermovement jump biomechanics in children with congenital clubfoot who have been surgically treated. This study will attempt to understand how children with CF perform CVJ. Information about biomechanics of the locomotion in children with clubfoot is important to choose the adequate stimuli to be applied during physical activity for those children, enhancing functional rehabilitation.

\section{Method}

\section{Participants}

Participants were 14 children with idiopathic CF (seven unilateral CF, $6.8 \pm 0.9$ years old, $30.0 \pm 5.1 \mathrm{~kg}$ mass, $1.30 \pm 0.05$ $\mathrm{m}$ tall and seven bilateral CF, $7.4 \pm 1.1$ years old, $34.4 \pm 11.0$ $\mathrm{kg}$ mass, $1.32 \pm 0.07 \mathrm{~m}$ tall) who were submitted to orthopedic surgery. Those children had no musculoskeletal lesions in the previous six months, foot pain or any functional difficulties in performing the daily activities. The surgical procedure was a stepwise release of posterior and medial structures of the foot and the Achilles tendon tenotomy. Control group consisted of 11 children $(7.6 \pm 0.7$ years old, $26.3 \pm 4.3 \mathrm{~kg}$ mass, $1.30 \pm 0.06$ $\mathrm{m}$ tall) without CF history and musculoskeletal lesions in the six months before the evaluation.

Ethical implications that involved the procedures in the study were approved by the Commission of Ethics in Research, and all information was explained to the children and their parents or guardians before the evaluation.

\section{Apparatus}

One Bertec force plate with strain gauge transducers (\#k80204, model 4060-15) was used to measure the vertical ground reaction force (GRF) at $1 \mathrm{kHz}$ sampling rate. The movements of the lower limbs at the sagittal plane were recorded with two video cameras (JVC-SVHS) at $50 \mathrm{~Hz}$ sampling rate (shutter 1:100), positioned to capture images in the sagittal plane of each leg of the children. A cube-shaped frame with sphericalmarkers at known distances from each other was used as a reference frame for the bidimensional reconstruction. Muscle activities were recorded by an electromyographical (EMG) system (1 $\mathrm{kHz}$ ) with an amplifier unit (100×) and active $\mathrm{Ag} / \mathrm{AgCh}$ bipolar surface electrodes $(11 \times)$.
APAS Ariel Dynamics software was used to digitalize and to calculate the bidimensional coordinates of the selected anatomical landmarks. Acknowledge software (Biopac System Inc, CA, USA) was used to synchronize GRF and EMG data. We used a manual trigger, which generated luminous signs to synchronize the kinematics and kinetics data.

\section{Procedure}

Passive reflective markers were attached to the participants with double-sided adhesive tape on the following anatomical points: the tuberosity of the fifth metatarsal bone, the lateral surface of the calcaneus, the distal apex of the lateral malleolus, the apex of the head of the fibula, the furthest distal point of the lateral femoral condyle, and greater femoral trochanter of the femur. Surface EMG electrodes were positioned from the belly of the medial head of the gastrocnemius and tibialis anterior muscles, based on SENIAN's recommendations ${ }^{10}$. A ground electrode was attached on the patella bone.

Participants were instructed to jump with their hands on the hips and with the trunk as erect as possible. In landing phase the subjects were instructed to flex the knees at $90^{\circ}$. Participants performed many trials before the measurements were taken. Children were asked to jump with maximal effort. Time flight was used to calculate the height of rise of the center of gravity.

Children stood on the forceplate, looking straight ahead, and after an voice command they performed the CVJ (Figure 1). Five trials were captured.

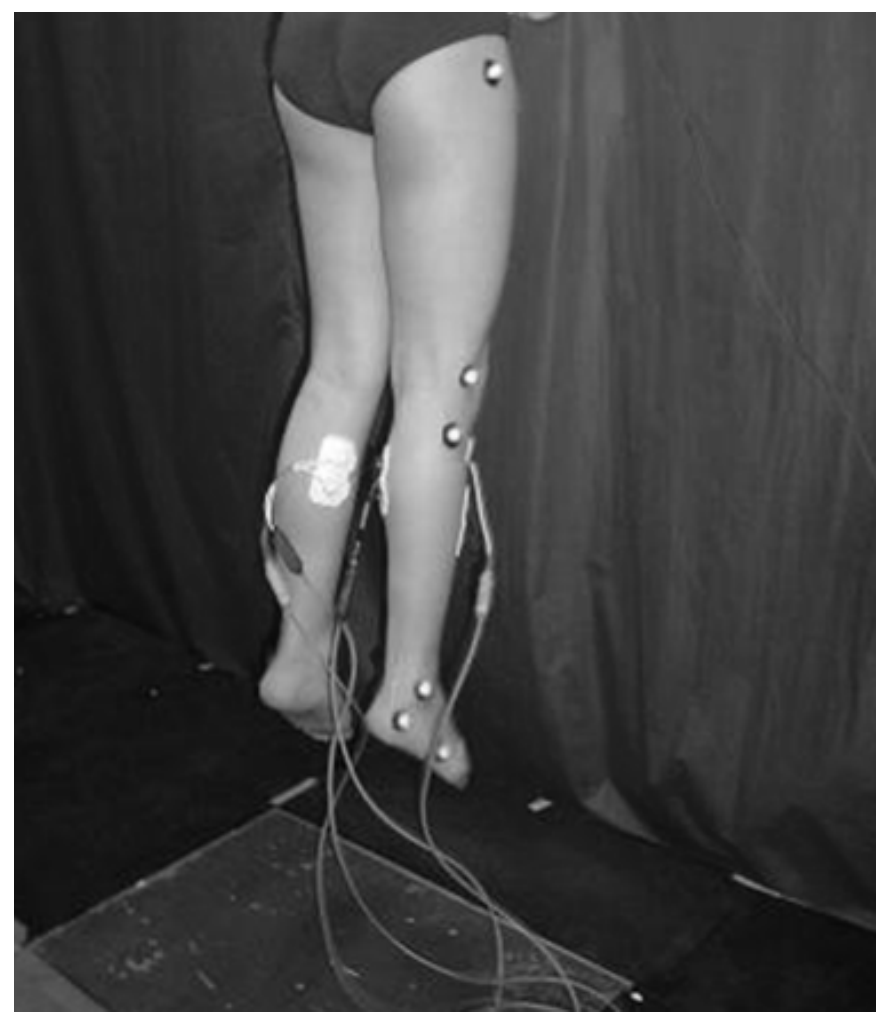

Figure 1 Countermovement vertical jump. 


\section{Data Analysis}

Preparation phase and landing phase of CVJ were analyzed. Kinetic raw data was filtered (second order Butterworth low-pass of $200 \mathrm{~Hz}$ ) and normalized by body weight. EMG data was filtered (second order Butterworth band-pass: $20-400 \mathrm{~Hz}$, and a notch filter: $60 \mathrm{~Hz}$ ), demeaned and normalized by the peak in both phases analyzed. Kinematics raw data was filtered (second order Butterworth low-pass of 10 $\mathrm{Hz}$ ). For all these calculations, Matlab 6.5 codes were used (The Mathworks Inc.).

\section{Variables and Statistical Analysis}

Variables were separated according to each leg for children with bilateral involvement and the affected and unaffected side for children with unilateral CF. In addition, we selected the dominant side of children in the control group. To evaluate results, statistical analyzes were performed according to the following classification: 1) control group; 2) bilateral CF group-right side; 3) bilateral CF group-left side; 4) unilateral CF group - affected side; 5) unilateral CF groupunaffected side.

A Friedman test was run to confirm that the trial variability effect could be neglected. Then, Wilcoxon test was used to verify whether the right and left were different. Kruskal-Wallis test was used to identify possible differences between the groups and post-hoc Mann-Whitney test was performed to compare differences between variables, which are described in full detail in the results.

For GRF, we calculated the following variables: GRF peak during the preparation phase (F1); GRF peak during the landing phase (F2); loading rate ( $\mathrm{LR}=\mathrm{F} 1$ divided by the time to reach $\mathrm{F} 1$ ). In addition, the jump height was calculated by the period between takeoff and contact after flight was used as the time in the air during the flight phase of the jump.

For angular kinematics, we calculated the following: knee flexion peak angle (kneel) and ankle dorsiflexion peak angle (ankle1) during the preparation phase; the knee (knee2) and ankle (ankle2) angles at the end of the preparation phase; knee (knee3) and ankle (ankle3) angles at the beginning of the landing phase; and the maximum knee flexion (knee4); and ankle dorsiflexion (ankle4) angles during the landing phase. For EMG, we calculated the gastrocnemius medialis and tibialis anterior muscles intensities during the preparation (GM1 and TA1) and the landing (GM2 and TA2) phases. Those variables are illustrated in Figure 2.

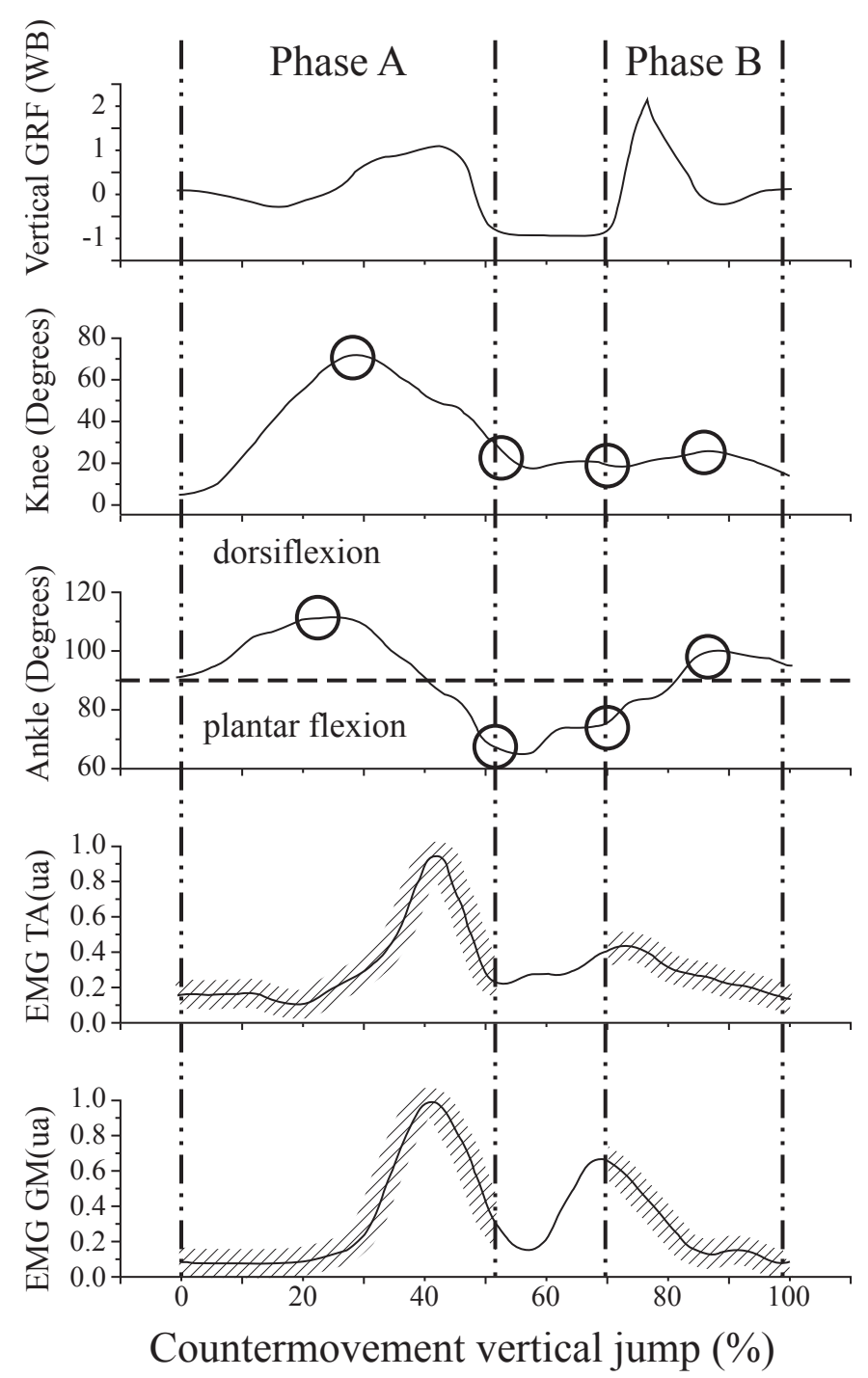

Figure 2 Parameters illustration of countermovement vertical jump data. Phase A: the preparation phase; Phase B: the landing phase. Circles indicate angular data; Shaded regions indicate muscle intensity data evaluated for gastrocnemius medialis $(\mathrm{GM})$ and tibialis anterior (TA).

\section{Results}

Jump height showed better performance for control group compared with the other groups $(p=0.02)$. These findings may be justified by biomechanical findings in the preparation phase described below.

During the preparation phase, for the maximum dorsiflexion, bilateral and unilateral (only the affected side) CF groups produced the smallest angle (Kruskal-Wallis $\mathrm{chi}^{2}=18.8$; d.f. $=$ $213 ; p=0.001)$; and between the unilateral groups, the affected side group presented the lowest angular ankle values (ankle1 variable). For F1, unilateral CF groups presented the lowest peaks (Kruskal-Wallis chi $^{2}=7.8$; d.f. $=122 ; \mathrm{p}=0.01$ ). For EMG, the lowest gastrocnemius medialis muscle activation (GM1) was found at the affected side of the unilateral $\mathrm{CF}$ group (KruskalWallis $\mathrm{chi}^{2}=12.6$; d.f. $=203 ; \mathrm{p}=0.01$ ). 
At the end of the preparation phase, before the aerial phase, $\mathrm{CF}$ groups showed the smallest plantarflexion, with a tendency to normal values in the non-affected side of the unilateral $\mathrm{CF}$ group (ankle2; Kruskal-Wallis $\mathrm{chi}^{2}=81.2$; d.f. $=213$; $\mathrm{p}<0.001$ ). The unilateral CF groups presented the largest knee angle values (knee2; Kruskal-Wallis $\mathrm{chi}^{2}=11.1$; d.f. $=213$; $\mathrm{p}=0.02$ ).

In the landing phase, the unilateral CF group flexed the knee more than the control group (Kruskal-Wallis $\mathrm{chi}^{2}=15.4$; d.f. $=213 ; p=0.004)$. The bilateral groups and the affected side of unilateral $\mathrm{CF}$ groups presented less plantar flexion compared to the unaffected side of unilateral $\mathrm{CF}$ and control groups (ankle3; Kruskal-Wallis $\mathrm{chi}^{2}=41.8$; d.f. $=213$; $\mathrm{p}<$ $0.001)$. For LR values, the CF groups were greater than the control (Kruskal-Wallis $\mathrm{chi}^{2}=12.8$; d.f. $=122 ; \mathrm{p}=0.002$ ). For EMG, the unaffected side CF group presented the highest the gastrocnemius muscle activity (GM2; Kruskal-Wallis chi ${ }^{2}$ $=37.5$; d.f. $=203 ; \mathrm{p}<0.001)$. For dorsiflexion angle (ankle4), lower values were found for all CF groups, and the unaffected side of the unilateral CF was similar to the control (KruskalWallis $\mathrm{chi}^{2}=14.15$; d.f. $\left.=213 ; \mathrm{p}=0.007\right)$.

Table 1 Comparison of the vertical ground reaction force parameters between the groups.

\begin{tabular}{|c|c|c|c|c|c|c|}
\hline Variables & Groups & Mean & Median & Standard Deviation & $\mathbf{N}$ & p-value \\
\hline \multirow{3}{*}{$\begin{array}{l}\text { F1* } \\
\text { (body weight) }\end{array}$} & 1 & 1.46 & 1.30 & 0.62 & 48 & \multirow{3}{*}{0.019} \\
\hline & 2 & 1.55 & 1.41 & 0.44 & 27 & \\
\hline & 3 & 1.22 & 1.20 & 0.42 & 24 & \\
\hline \multirow{3}{*}{$\begin{array}{l}\mathrm{LR}^{+} \\
(\mathrm{PC} / \mathrm{s})\end{array}$} & 1 & 40.46 & 30.45 & 30.84 & 48 & \multirow{3}{*}{0.002} \\
\hline & 2 & 63.22 & 44.88 & 39.92 & 27 & \\
\hline & 3 & 58.65 & 49.93 & 41.78 & 24 & \\
\hline \multirow{3}{*}{$\begin{array}{l}\text { F2 } \\
\text { (body weight) }\end{array}$} & 1 & 2.89 & 2.51 & 1.61 & 48 & \multirow{3}{*}{0.095} \\
\hline & 2 & 3.29 & 2.84 & 1.41 & 27 & \\
\hline & 3 & 3.35 & 3.20 & 1.18 & 24 & \\
\hline
\end{tabular}

1) Control group; 2) Bilateral clubfoot group; 3) Unilateral clubfoot group. p $£ 0.05$.

$* 3<1 ;+1<2,3$

Table 2 Comparison of the angular knee parameters calculated between the groups.

\begin{tabular}{|c|c|c|c|c|c|c|}
\hline Variables & Groups & Mean & Median & Standard Deviation & $\mathbf{N}$ & p-value \\
\hline \multirow{5}{*}{$\begin{array}{l}\text { knee1 } \\
\text { (Degrees) }\end{array}$} & 1 & 78.10 & 76.36 & 10.16 & 110 & \multirow{5}{*}{0.069} \\
\hline & 2 & 72.31 & 71.49 & 15.76 & 27 & \\
\hline & 3 & 72.03 & 70.66 & 18.05 & 27 & \\
\hline & 4 & 76.88 & 75.93 & 9.35 & 25 & \\
\hline & 5 & 77.16 & 78.20 & 10.53 & 25 & \\
\hline \multirow{5}{*}{$\begin{array}{l}\text { knee2\# } \\
\text { (Degrees) }\end{array}$} & 1 & 3.20 & 2.10 & 2.79 & 110 & \multirow{5}{*}{0.025} \\
\hline & 2 & 5.16 & 4.27 & 4.36 & 27 & \\
\hline & 3 & 4.69 & 2.50 & 4.97 & 27 & \\
\hline & 4 & 6.22 & 6.03 & 4.36 & 25 & \\
\hline & 5 & 5.20 & 3.98 & 4.83 & 25 & \\
\hline \multirow{5}{*}{$\begin{array}{l}\text { knee3t } \\
\text { (Degrees) }\end{array}$} & 1 & 6.96 & 5.14 & 7.72 & 110 & \multirow{5}{*}{0.004} \\
\hline & 2 & 7.09 & 7.02 & 4.81 & 27 & \\
\hline & 3 & 8.14 & 5.33 & 7.69 & 27 & \\
\hline & 4 & 8.89 & 8.41 & 3.54 & 25 & \\
\hline & 5 & 9.40 & 8.85 & 4.91 & 25 & \\
\hline
\end{tabular}


...continuation

\begin{tabular}{|c|c|c|c|c|c|c|}
\hline \multirow{5}{*}{$\begin{array}{l}\text { knee } 4 \\
\text { (Degrees) }\end{array}$} & 1 & 54.95 & 52.39 & 18.82 & 110 & \multirow{5}{*}{0.840} \\
\hline & 2 & 51.72 & 51.21 & 13.69 & 27 & \\
\hline & 3 & 51.87 & 53.09 & 17.07 & 27 & \\
\hline & 4 & 51.99 & 50.97 & 16.20 & 25 & \\
\hline & 5 & 55.92 & 55.02 & 15.91 & 25 & \\
\hline
\end{tabular}

1) Control group; 2) Bilateral CF group, right side; 3) Bilateral CF group, left side; 4) Unilateral CF group, affected side; 5) Unilateral CF group, unaffected side. $\mathrm{p} £ 0.05$.

$\# 1<4,5 ;+1<4,5$

Table 3 Comparison of the angular ankle parameters calculated between the groups.

\begin{tabular}{|c|c|c|c|c|c|c|}
\hline Variables & Groups & Mean & Median & $\begin{array}{l}\text { Standard } \\
\text { Deviation }\end{array}$ & $\mathbf{N}$ & p-value \\
\hline \multirow{5}{*}{$\begin{array}{l}\text { ankle1 } 1^{* ; * *} \\
\text { (Degrees) }\end{array}$} & 1 & 114.08 & 114.80 & 8.97 & 110 & \multirow{5}{*}{0.001} \\
\hline & 2 & 106.24 & 109.26 & 13.23 & 27 & \\
\hline & 3 & 107.97 & 112.03 & 13.19 & 27 & \\
\hline & 4 & 104.54 & 109.24 & 11.36 & 25 & \\
\hline & 5 & 113.16 & 113.14 & 5.80 & 25 & \\
\hline \multirow{5}{*}{$\begin{array}{l}\text { ankle2\# } \\
\text { (Degrees) }\end{array}$} & 1 & 43.08 & 43.46 & 7.39 & 110 & \multirow{5}{*}{$<0.001$} \\
\hline & 2 & 61.54 & 64.29 & 7.67 & 27 & \\
\hline & 3 & 59.51 & 61.66 & 8.60 & 27 & \\
\hline & 4 & 59.35 & 50.19 & 17.88 & 25 & \\
\hline & 5 & 50.55 & 47.94 & 16.84 & 25 & \\
\hline \multirow{5}{*}{$\begin{array}{l}\text { ankle3t } \\
\text { (Degrees) }\end{array}$} & 1 & 53.74 & 52.82 & 9.76 & 110 & \multirow{5}{*}{$<0.001$} \\
\hline & 2 & 66.50 & 68.86 & 9.66 & 27 & \\
\hline & 3 & 63.61 & 66.99 & 11.61 & 27 & \\
\hline & 4 & 67.38 & 58.32 & 17.81 & 25 & \\
\hline & 5 & 58.76 & 55.26 & 15.62 & 25 & \\
\hline \multirow{5}{*}{$\begin{array}{l}\text { ankle4H } \\
\text { (Degrees) }\end{array}$} & 1 & 110.17 & 109.85 & 8.13 & 110 & \multirow{5}{*}{0.007} \\
\hline & 2 & 105.05 & 103.49 & 10.93 & 27 & \\
\hline & 3 & 105.29 & 106.47 & 9.89 & 27 & \\
\hline & 4 & 102.68 & 102.33 & 11.42 & 25 & \\
\hline & 5 & 108.80 & 109.67 & 6.90 & 25 & \\
\hline
\end{tabular}

1) Control group; 2) Bilateral CF group, right side; 3) Bilateral CF group, left side; 4) Unilateral CF group, affected side; 5) Unilateral CF group, unaffected side. $\mathrm{p} £ 0.05$. ( $90^{\circ}$ is considered the neutral position; the smaller the angle is greater plantar flexion).

${ }^{*} 1>4,3,2 ;{ }^{* *} 5>4 ; \# 1<2,3,4,5 ;+1<2,3,4 ; H_{1,5}<2,3,4$

\section{Discussion}

Extensive studies have surveyed children's locomotion by investigating the biomechanics of walking ${ }^{2-5}$. However, little
Table 4 Comparison for gastrocnemius medialis (GM) and tibialis anterior (TA) muscles calculated between the groups.

\begin{tabular}{|c|c|c|c|c|c|c|}
\hline Variables & Groups & Mean & Median & $\begin{array}{l}\text { Standard } \\
\text { Deviation }\end{array}$ & $\mathbf{N}$ & p-value \\
\hline \multirow{5}{*}{$\begin{array}{l}\text { GM1* } \\
\text { (ua) }\end{array}$} & 1 & 0.100 & 0.095 & 0.029 & 98 & \multirow{5}{*}{0.013} \\
\hline & 2 & 0.092 & 0.093 & 0.017 & 29 & \\
\hline & 3 & 0.126 & 0.093 & 0.068 & 29 & \\
\hline & 4 & 0.082 & 0.076 & 0.030 & 24 & \\
\hline & 5 & 0.097 & 0.094 & 0.018 & 24 & \\
\hline \multirow{5}{*}{$\begin{array}{l}\text { GM2\# } \\
\text { (ua) }\end{array}$} & 1 & 0.092 & 0.082 & 0.033 & 98 & \multirow{5}{*}{$<0.001$} \\
\hline & 2 & 0.117 & 0.113 & 0.028 & 29 & \\
\hline & 3 & 0.107 & 0.095 & 0.046 & 29 & \\
\hline & 4 & 0.091 & 0.082 & 0.041 & 24 & \\
\hline & 5 & 0.155 & 0.161 & 0.050 & 24 & \\
\hline \multirow{5}{*}{$\begin{array}{l}\text { TA1 } \\
\text { (ua) }\end{array}$} & 1 & 0.129 & 0.130 & 0.033 & 98 & \multirow{5}{*}{0.752} \\
\hline & 2 & 0.123 & 0.129 & 0.039 & 29 & \\
\hline & 3 & 0.121 & 0.122 & 0.038 & 29 & \\
\hline & 4 & 0.129 & 0.122 & 0.030 & 24 & \\
\hline & 5 & 0.124 & 0.119 & 0.039 & 24 & \\
\hline \multirow{5}{*}{$\begin{array}{l}\text { TA2 } \\
\text { (ua) }\end{array}$} & 1 & 0.136 & 0.135 & 0.037 & 98 & \multirow{5}{*}{0.739} \\
\hline & 2 & 0.132 & 0.127 & 0.048 & 29 & \\
\hline & 3 & 0.126 & 0.123 & 0.033 & 29 & \\
\hline & 4 & 0.147 & 0.135 & 0.054 & 24 & \\
\hline & 5 & 0.134 & 0.139 & 0.039 & 24 & \\
\hline
\end{tabular}

1) Control group; 2) Bilateral $\mathrm{CF}$ group, right side; 3) Bilateral $\mathrm{CF}$ group, left side; 4) Unilateral CF group, affected side; 5) Unilateral CF group, unaffected side. $\mathrm{p}<0.05$.

${ }^{*} 4<1,2,3,5$; $\# 5>1,2,3,4$.

scientific study has studied other functional abilities. The vertical jump, for example, is an important functional activity that is commonly applied in training and rehabilitation ${ }^{11}$. Mechanical loading during childhood, based on jumping activities, for 
example, plays a critical role in normal growth and development of the skeleton ${ }^{7}$. The lack of understanding about the relationship among biomechanical patterns in children with pathological situations, during functional activities, is an obstacle to our understanding of prophylactic exercises and the best rehabilitation. The descriptions of these characteristics in pathological conditions are poor. The objective of this study was to evaluate and understand the functional adaptations that can be described for the CF population. It may be a useful basis for obtaining a functional quantitative evaluation of clubfooted children who have had surgery.

In preparation for the $\mathrm{CVJ}$ that demands high angular amplitude $^{11}$, CF children had a limitation in ankle movement that can be caused by the residual stiffness within the foot that is characteristic of the pathology's evolution. This restriction on movement was used by Davies, Kiefer, Zernicke ${ }^{2}$ to explain, in gait analysis, reduced plantarflexion during push-off for $\mathrm{CF}$ groups. At the end of the preparation phase there occurred a reduction of plantar flexion and a greater knee flexion in all CF groups. Less gastrocnemius muscular activation in the affected side of the unilateral CF group was found in this stage. These findings show that inefficient movement in the preparation phase, demonstrated by an abnormal angular movement and muscular activity, can contribute to the lower GRF in the propulsive phase, characterized in this study by the CF children. These characteristics are similar to gait parameters, which presented plantar flexion differences during push-off, limitation of ankle dorsiflexion and weakness of the gastrocnemius muscle ${ }^{2,4}$.

In the beginning of the landing phase, the CF groups presented angular ankle alteration in all groups and knee flexion alteration in the unilateral CF groups. CF children presented, in addition, a larger rate of loading to the peak of vertical GRF. The ability to control forces during landing is key to preventing injuries. The literature shows that landing with a great impact force, characterized by a bigger rate of loading, may be a risk factor for lower limbs ${ }^{12-13}$. The increased impact forces could be the result of angular alteration in the foot's initial contact with the ground ${ }^{13}$, found in our study.

Gastrocnemius activity is fundamental to better ankle joint deceleration in the landing phase ${ }^{6}$. In this study, the hypothesis was that this muscle activity alteration would have been presented in both CF groups because they did a tenotomy of the Achilles tendon. This hypothesis was not proven, probably because the surgical procedure was done in the first year of life. However, we found disequilibrium in gastrocnemius muscular activation in the unaffected side of the unilateral $\mathrm{CF}$ group. Therefore, this lower limb needs to compensate the structural unbalance with great muscular activity. Davies, Kiefer, Zernicke ${ }^{2}$ suggested that this contralateral limb may have compensated for insufficient motion of the affected side.

We found no unbalance in the biomechanical parameters analyzed between the sides of the bilateral CF groups. These results can be hypothesized by an adjustment of the body's symmetry in dynamic situations that can make changes in motor control, with central nervous system adaptation. The unilateral $\mathrm{CF}$ children demonstrated unbalance between the affected and unaffected sides, with differences when compared with the control group. No significant differences had been evidenced in GRF parameters between the clubfoot and the contralateral limb of the children with unilateral clubfoot in the studies by Davies, Kiefer, Zernicke². Favre, Exner, Drerup, Schimid, Wetz , Jabob ${ }^{5}$ identified similar differences under the unaffected foot of unilateral clubfeet and the normal foot in pressure parameters during gait analysis. It is supposed that these differences found in this study cause asymmetries and generate mainly a potential risk to the muscular-skeletal system. The other limitation of this study is that we used only one forceplate, which makes a GRF parameter comparison between the sides impossible. Based on the fact that there were differences between kinematic and electromyographical data, if we had used two plateforces the GRF parameter results would likely have followed the same differences.

\section{Conclusion}

The smallest dorsiflexion angles in the preparation phase and the lowest GRF in the propulsive phase contribute to inefficient $\mathrm{CVJ}$ in CF children. In addition, the great impact force in landing phase was associated with a poor mechanism of angle control.

The results presented in this study could be an incentive for specific exercises, which contribute to preventive conduct for this population. Future functional activity investigations should use research methodologies to quantify internal forces in $\mathrm{CF}$ children who have undergone different surgical and conservative treatment techniques.

\section{References}

1. Barker S, Chesney D, Miedzybrodzka Z, Maffulli N. Genetics and epidemiology of idiopathic congenital talipes equinovarus. J Pediatr Orthop. 2003;23(2):265-72.

2. Davies TC, Kiefer G, Zernicke RF. Kinematics and kinetics of the hip, knee, and ankle of children with clubfoot after posteromedial release. J. Pediatr. Orthop. 2001; 21:366-371.

3. Beyaert C, Haumont T, Paysant J, Lascombes P, Andre JM. The effect of inturning of the foot on knee kinematics and kinetics in children with treated idiopathic clubfoot. Clin. Biomech. 2003; 18: 670-676.

4. Theologis TN, Harrington ME, Thompson N, Benson MK. Dynamic foot movement in children treated for congenital talipes equinovarus. J. Bone Joint. Surg. 2003; 85:572-577.

5. Favre P, Exner GU, DrerupB, Schimid D, Wetz HH, Jabob HA. The contralateral foot in children with unilateral clubfoot: a study of pressures and forces involved in gait. J. Pediatr. Orthop. 2007; 27:54-59.

6. Walsh MS, Bohm H, Butterfield MM, Santhosam J. Gender bias in the effects of arms and countermovement on jumping performance. J. Strength. Cond. Res. 2007; 21:362-366.McKay H, Tsang G, Heinonem A, Mackelvie K, Sanderson D, Khan KM. Ground reaction forces associated with an effective elementary school based jumping intervention. Br. J. Sports Med. 2005; 39:10-14. 
7. Pain MTG, Challis JH. The influence of soft tissue movement on ground reaction forces, joint torques and joint reaction forces in drop landing. J. Biomech. 2006; 39:119-124.

8. Caulfield B, Garrett M. Changes in ground reaction force during jump landing in subjects with functional instability of the ankle joint. Clin. Biomech. 2004; 19:617-621.

9. Hermens HJ, Freriks B, Disselhorst-Klug C, Rau G. Development of recommendations for SEMG sensors and sensor placement procedures. J. Electromyogr. Kinesiol. 2000; 10:361-374.

10. Swartz EE, Decoster LC, Russell PJ, Croce RV. Effects of developmental stage and sex on lower extremity kinematics and vertical ground reaction forces during landing. J. Athl. Train. 2005; 40:9-14.

11. Chappell JD, Herman DC, Knight BS, Kirkendall DT, Garrett WE Yu. B. Effect of fatigue on knee kinetics and kinematics in stop-jump tasks. Am. J. Sports Med. 2005; 33:1022-1029.

12. Yu B, Lin CF, Garrett WE. Lower extremity biomechanics during the landing of a stop-jump task. Clin. Biomech. 2006; 21:297-305.

\section{Acknowledgments}

Financial support for this research came from CAPES Brazilian Funding Agency.

\section{Corresponding author}

Renato José Soares

University of Taubaté, UNITAU. School of Physical Therapy. Rua Marechal Arthur da Costa e Silva, 1055. 12010-490. Taubaté/SP, Brazil

Email address: renato.soares@unitau.com.br

Manuscript received on May 05, 2016

Manuscript accepted on August 21, 2016

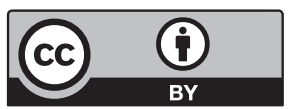

Motriz. The Journal of Physical Education. UNESP. Rio Claro, SP, Brazil - eISSN: 1980-6574 - under a license Creative Commons - Version 3.0 